\title{
Contribution of Nutrition to Child Health
}

\author{
Benjamin Caballero \\ Center for Human Nutrition, J ohns Hopkins Universtiy, Baltimore, Md., USA
}

This supplement presents the proceedings of the workshop on nutrition and child health, which was held as part of the 17th International Congress of Nutrition, held in Vienna in August of 2001. The workshop offered the opportunity to review some of the key dietary and nutritional factors affecting child health worldwide, and once more emphasized the importance of the global context for the health professional practicing in today's world. The fact that nutritional and dietary factors account, directly or indirectly, for 6 out of every 10 deaths in infancy and childhood further underscores the importance of this topic.

Current global trends in child health indicate modest, but consistent progress in reducing infant mortality and malnutrition in most regions of the world, with the exception of Sub-Saharan Africa. But a new challenge is emerging from this progress: ensuring a good quality of life to surviving children. First, there is increasing evidence that chronic malnutrition ('stunting') early in life is associated with reduced school performance, and ultimately with higher disease and mortality risks in adult life. Similarly, early micronutrient deficiencies, for example of iron and calcium, may have a sustained impact on the health of adolescents and adults.

Dr. Francesco Branca, from the National Institute of Nutrition in Rome, discusses the impact of micronutrient deficiencies on growth. The evidence from studies in deficient populations, combined with advances in our understanding of the biological role of nutrients in healthy chil- dren, has placed micronutrient deficiencies at the forefront of our efforts to optimize growth. Recent studies have shown that even moderate single-nutrient deficiencies, if occurring at critical periods of child development, may have a long-term impact on health. For example, marginal calcium intake during childhood may result in a deficient bone mineral accumulation at the end of the growth period, resulting in a substantial increase in the risk of osteoporosis after menopause. Similarly, some data suggest that iron deficiency in early years, even when corrected, may result in persistently lower scores in several psychomotor tests years later.

Food preferences and eating behaviors during childhood are another important determinant of nutritional status. This area was reviewed by Prof. Joachim Westenhoefer, of the University of Applied Science in Hamburg. He described a constant interaction between innate and acquired taste preferences, and discussed biological selfregulation and its interaction with environmental factors as the infant develops. This process, conditioned by exposure, social influences, and associative learning, is a key element in our ability to positively influence children's ingestive behavior. The sociocultural context of food consumption in modern society raises a concern about its impact on young children, who receive conflicting messages promoting food consumption as well as thinness.

Although generally recognized as important, the interactions between socioeconomic level and nutritional status are not easy to document. Dr. Michael Murphy dis-

\section{KARGER \\ Fax +41613061234 \\ E-Mail karger@karger.ch \\ www.karger.com

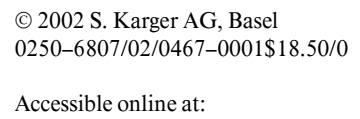

Benjamin Caballero

Center for Human Nutrition, Johns Hopkins University

615 N. Wolfe St.

Baltimore, MD 21205 (USA)

Tel. +1 410614 4070, Fax +1 410955 0195, E-Mail caballero@jhu.edu 
cussed this important issue, presenting evidence of the significant impact that social and economic factors have in the dietary intake of children in developed societies. He also described a program to improve nutrient intake by targeting elementary schools in deprived areas. The positive effect of increasing nutritional intake via school breakfast programs on school performance and attendance are evident from the data presented.

There is a great deal of variability in the degree to which children and adolescents fulfill their nutrient requirements, even in industrialized societies. European surveys, summarized by Prof. Lluis Serra-Majem, indicate that children and adolescents are the population groups most likely to have higher risk of nutritional deficiencies, particularly for iron, vitamin $\mathrm{C}, \mathrm{E}, \mathrm{B}_{6}$ and folates. Factors affecting nutrient intake in children are related to income level, parental level of education and rural location, among others. Most surveys in industrialized countries coincide in pointing to the important contribution of food fortification for providing adequate micronutrient intake for the pediatric age population.
In summary, there is encouraging progress in reducing the global burden of undernutrition in children. At the same time, our enthusiasm is tempered by the challenge of providing adequate nutrition to children who survive the early years, and we are more aware of the long-term effects of transient nutritional deficiencies at critical periods of development. Promotion of healthy eating habits early on must receive much more attention, and healthcare professionals should recognize this aspect as a potentially very effective opportunity to improve nutritional status via parental education. Finally, as we learn more about the specific nutrient needs of infants, children and adolescents, we will have new opportunities to improve the micronutrient content of foods and to target our efforts to specific age groups and phases of growth. The phrase 'the child is the father of the future' could not be more appropriate to describe the enormous importance of nutrition and health during the early years of life.

Finally, on behalf of the workshop's participants, I would like to acknowledge the support of Kellogg for its organization. 